\title{
Kidney Disease Predictor Based on Medical Decision Support System
}

\author{
Ebtihal Abuobaid ${ }^{1}$, Sameeha Ahmad Al-Shelleh ${ }^{2}$, Bahaaldeen Alshraideh ${ }^{3}$, Abedalrahman Alshraideh ${ }^{4}$ and \\ Mohammad Alshraideh ${ }^{5 *}$ \\ 1,5 Department of computer science, The University of Jordan, Jordan \\ ${ }^{2,3,4}$ Medicine School, The University of Jordan, Jordan
}

Submission: November 13, 2020; Published: December 14, 2020

*Corresponding author: Mohammad Alshraideh, Department of computer science, The University of Jordan, Jordan

\begin{abstract}
Renal failure will increase mortality if untreated. When kidneys fail, the buildup of toxins occurs. Which affects the whole body and cause complications. There are numerous causes for renal failure, but we evaluate its main causes, which are Hypertension, Diabetes, Glomerulo Nephritis, Vesicoureteral Reflux and Polycystic Kidney Disease. Kidneys, in general, are very complicated organs. Nevertheless, most kidney diseases share a lot of presenting symptoms, which may lead to some delay in medical diagnosis. This study aims to develop a decision support system to predict the main cause of renal failure in patients using their memory, making quick predictions that may aid in the final diagnosis. A multilayer perceptron (MLP) feed-forward neural network was proposed in this research. The input layer of the proposed system included 32 input variables. An iterative process was used to determine the number of neurons and hidden layers. Furthermore, a resilient backpropagation algorithm (Rprop) was used to train the system. In order to access the generalization of the proposed system, a 10-fold cross-validation scheme was used. We obtained an encouraging result for prediction patient from the experiments made on the data that were taken from 180 patients' medical records at seven hospitals in Jordan.
\end{abstract}

Keywords: Kidney Disease; Kidney Failure; Prediction of Kidney failure; Clinical Decision Support System

\section{Introduction}

According to the Hashemite Kingdom of Jordan Ministry of Health Focal Point for Health Information, and according to the national list for local renal failure that there is about new 300 yearly cases are added to this list in Jordan (Prime Ministry). Significant lifesaving can be achieved if an accurate diagnosis can be made for patients suffering from various kidney diseases. And because kidney diseases symptoms can be similar, an accurate diagnosis cannot be an easy task.

The kidneys are vital to major organs to keep the balance in the whole body, so talking about renal failure will implicate talking about the more systemic effect on the whole body with major resultant systemic complications too.

Artificial neural network (ANN) field has gained its momentum in almost any domain of research and just recently has become a reliable tool in the medical domain [1-5]. ANN can help in solving diagnostic and prognostic problems in a variety of medical domains, by providing useful methods, techniques and tools. It is well suited to specialized hospitals and clinics, because of many new cases entered daily. With the data, symptoms and diagnosis are added, ANN could be applied on that data to help in the prediction of disease progression, the extraction of medical knowledge for outcomes research, for therapy planning and support, and overall patient management. It can be used for data analysis, such as detection of regularities in the data by appropriately dealing with imperfect data, interpretation of continuous data used in the Intensive Care Unit, and for intelligent alarming resulting in effective and efficient monitoring. ANN systems are very successful in the healthcare environment, due to its enhancement of medical experts work and improvement of the efficiency and quality of medical care.

To reduce the diagnosis time and to improve its accuracy, a powerful medical decision support system (MDSS) has been developed. A Multilayer Perceptron (MLP) Feed-Forward Neural Network is used in developing the system to diagnosis the six main renal failure cause diseases.

Multiple experiments are done with various inputs between $30-32$ input variables. Whereas, the output layer contains one neuron, which represents one disease causes based on the patient 
case. In order to access the generalization of the proposed system, a 10 -fold cross-validation scheme is used. t. The data was taken from 180 patients' questionnaires, who are suffering from renal failure because of one of the six causes. These questionnaires are collected from seven different hospitals in The Hashemite Kingdom of Jordan.

As it is known, the medical diagnosis by nature is a complex and fuzzy cognitive process, and soft computing methods such as neural network have been widely used in solving these medical problems. In Self Organization Maps for prediction of kidney dysfunction by Ali [6], in this paper, he used the Kohonen- SOM network as a prediction for kidney dysfunction. The peculiar about Kohonen networks is that they are consist of two layers, input and output layer. The output layer can be two-diminutions. This system works as follows, first, it initializes the input nodes, the output nodes and connection weights. Then describe each set-in order ( $\mathrm{N}$ coordination). After that, it computes the distance of all nodes. Finally, find the winning distance which will be the minimum one. All these steps will be according to specific mathematic calculations that were added to that paper. In a multilayer perceptron - based medical decision support system for heart disease diagnosis by Qatawneh et al. [2] they used a neural network to develop a medical decision support system to support the diagnosis Venous Thromboembolism Risk Classification, Applied Computing and Informatics.

The computational model in this paper based on multilayer perceptron network. This model is used consist of 3 layers: an input layer, a hidden layer, and output layer. The input layer takes 40 variables, the number of nodes in the hidden layer is determined through the cascade learning process and with an output of 5 nodes corresponding to the heart diseases. This system is applied to a large number of patient cases, that prove at the end that the system has a strong capability to classify the 5 heart diseases with $>90$ accuracies. Another paper is Using Artificial Neural Network to Predict Cirrhosis in Patients with Chronic Hepatitis B Infection with Seven Routine Laboratory Findings by Vahdani [7]. The data on this system was obtained by taking specific tests and liver biopsies from all patients. According to that, liver diseases was obtained. Backpropagation and ANN analysis were used to train the data. In this model, there were 8 neurons for input, 15 neurons in the middle, and 1 neuron for output. The important thing about this paper is that the data were divided into 2 groups training and testing with two thirds and one third for them respectively. And multiple logistic regression models are applied to the training group and performed on the test group to allow prediction. Turkoglu et al. [8] presented an expert diagnosis system for the interpretation of the Doppler signals of the heart valve diseases using a back-propagation neural network. The test results showed that this system was effective to detect Doppler heart sounds. The correct classification rate was about $94 \%$ for normal subjects and 95.9\% for abnormal subjects. All these studies depend on specific tests that the patient goes throw to use with the computerized system to have a specific decision. On our paper, on the other hand, it depends on the patient's memory to have an approximate decision to start with.

\section{Background and Related Works}

Multilayer Perceptron is one of the most frequently used neural network models due to its clear architecture and comparably simple algorithm. The multilayer perceptron system consists of 3 layers: one input layer, one or more hidden layers, one output layer. The multilayer perceptron is a feed-forward network, which means that each layer receives the input from the previous layer. In other words, the signals flow from the input to the first hidden layer forwarded to the next until finally reached to the output layer (hopefully). The feed-forward structures have proved most useful in solving non-linearly separable problems [9-14].

The process in Multilayer perceptron starts when the input layer serves the values of the input variables to the first (or the only) hidden layer. Then the hidden or the output layer units (depends on which layer is an intern to process) calculate its activation value by taking the weighted sum of the outputs of the units in the preceding layer. The activation value is passed through the activation function to produce the output of the neuron. When the process is executed, the last output (of the output layer) is the output of the whole process.

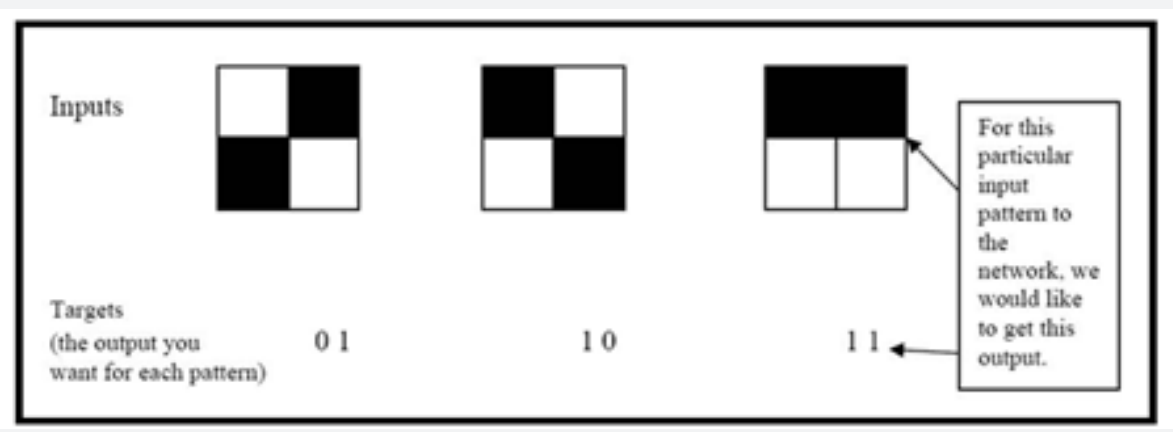

Figure 1: A Back Propagation Training Set [15]. 
MLP neural networks have been applied successfully to solve difficult and diverse problems by training them in a supervised manner with a highly popular algorithm known as Back Propagation which uses the data to adjust the network's weights and biases in a manner that minimizes the error in its predictions on the training set $[15,16]$. Back-Propagation is the training or learning algorithm rather than the network itself (Robert Gordon University) (Figure 1). To notice more about it lets consider the following example:

So, if we put in the first pattern to the network, we would like the output to be 01 as shown in next Figure (a black pixel is represented by 1 and a white by 0 ) (Figure 2). The input and its corresponding target are called a Training Pair. Once the network is trained, it will provide the desired output for any of the input patterns.

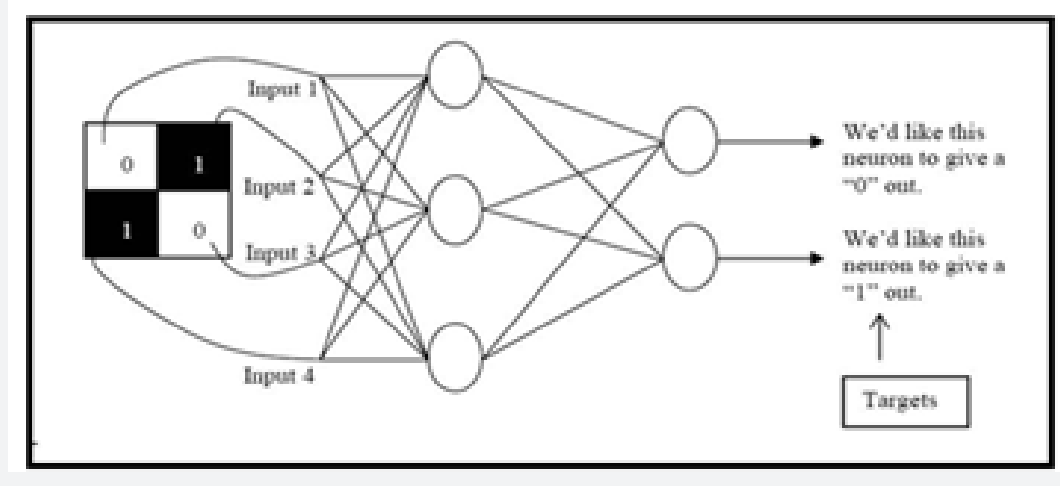

Figure 2: Applying a Training Pair to a Network [15].

A complementary learning fuzzy neural network was proposed in [1] for Ovarian cancer diagnosis. In [17-19] a modified fuzzy cellular neural network was proposed to effectively segment CT liver images, which will help in the early diagnosis of liver cancer. Adaptive Neuro-Fuzzy Inference System (ANFIS) is one of the intelligentsystems that showed promising performance in different aspects of our life, and more widely in medical applications. ANFIS has been implemented in many medical diagnoses such as human action recognition [20-23] and epilepsy seizure [16,24]. Contentbased image retrieval system, as a tool for discrimination between the normal and abnormal medical images, was developed in [25], heart valve diseases [26], rheumatoid arthritis [27,28], prostate cancer [7,29-32], and breast cancer [33]. ANFIS showed an overall accuracy in detecting glaucoma of $90.0 \%$ as reported in [33]. ANFIS illustrated a better performance in detecting four types of a brain tumour when compared with the performance of probabilistic neural network classifiers [34].

\section{System Architecture}

This paper is consisting of two systems, each with four different experiments; the first and the second systems are using linear activation function and TANH activation function as an output function respectively. And the main difference between their experiments is the input variables. These variations and different experiments are done to achieve higher classification accuracy, by adding or suspending some inputs. The input variables are between 30-32 inputs that are gathered from patients or patients' relatives depending on their remembrance.

These variables can be divided into five categories:

a) Basic information of a patient (including the age and the gender)

b) The patient's history (before dialyses)

c) The patient's family history

d) Symptoms

e) Physical examination

The questionnaire that is used was filled by patients. The survey consists of easy questions to normal people is specific and direct to the point. Most of the attributes were assigned to have a yes or no value to indicate the presence or absence of an attribute. Other attributes are not so they will be designated as follows:

a) Age when starting dialysis, vintage years on dialysis, and time of diagnosis of high blood pressure, all values are normalized into the range (0-1).

b) Hemoglobin $\mathrm{A} 1 \mathrm{C}$ test and daily blood sugar have three values (low, high, average).

\section{Input Variables Encoding Scheme}

Neural networks only deal with numerical values; therefore, the 32 (or 31,30 ) variables are encoded into numerical values using the following structure scheme: 
Age, number of years on dialysis, and time of diagnosis of high blood pressure are all are normalized into range (0-1).

a) Variables with two attributes are referred to 0 and 1 while 0 is for the absence of specific symptom and 1 for the appearance of that symptom. Also, 0 represents the male, 1 represent the female, in gender attribute.

b) Variables with 3 attributes like types of sugar test are referred to $-1,0,1$ where -1 represents the low term, 0 represents the average, and 1 represents the high term.

After encoding, the training dataset was standardized to have a zero mean and a unit standard deviation and based on the information from the training dataset during the standardization; the validation and test datasets were also normalized to have a zero mean and a unit standard deviation.

\section{Number of Hidden Layers and Hidden Neurons}

Because determining the number of hidden layers and hidden neurons in each layer in feedforward networks is one of the unresolved functions. So repeated process to figure the best number of hidden layers and neurons in each hidden layer is used. In the repeated process, a ten-fold cross-validation technique is used to access the generalization for each architecture. The whole process works as follow:

Step1: Start testing with one hidden layer; by applying the following equation to find the number of neurons in the first hidden layer:

$$
n_{f}=\left(n_{i}+n_{o}\right) / 2
$$

Where $n_{f}$ is the number of neurons in the first hidden layer, $n_{i}$ is the number of neurons in the input layer and no is the number of the neurons in the output layer. If $\mathrm{n}_{\mathrm{f}}$ was odd (not a fixed) number then apply the ceil and the floor operations, so you will get two values, and for more precise results take another number which is floor $\left(n_{\mathrm{f}}\right)-1$, so you will have three numbers of neurons in the first layer to start with.

Step 2: Add another layer; the number of neurons in this layer will be half the number of neurons in the previous layer.

Step 3: Repeat step two until the number of the hidden neurons in the layer is equal to one.

\section{Data Preparation}

In this paper, the renal failure because dataset used to test and train our systems is consisting of the total number of 180 cases. For six diseases, 30 cases for each, gathered from seven hospitals in Jordan:

Note that the 180 cases are taken from a total of 313 questionnaires, and it reduces to 180 due to the lake of cases in the VUS and GN which reach to 30 and 34 respectively.
This paper is consisting of two models, each with four different experiments; the first and the second models are using linear activation function and TANH activation function as an output function, respectively. And the main difference between their experiments is the input variables. These variations and different experiments are done to achieve higher classification accuracy, by adding or suspending some inputs. The input variables are between 30-32 inputs that are gathered from patients or patients' relatives depending on their remembrance.

These variables can be divided into five categories:

Basic information of a patient (including the age and the gender).

f) The patient's history (before dialyses).

g) Patients' family history.

h) Symptoms.

i) Physical examination.

To estimate the performance of the system, its accuracy and improve its generalization we used a technique called crossvalidation, it determines the accuracy by dividing the number of correct classifications by the overall number of records in the dataset (Yan et al., 2006).

This technique work by partitioning the dataset into training data, validation data and testing data, training data used to perform the analysis while testing data for test the analysis and validation data to avoid overfitting of the network [35-37].

In this paper we used 10 folds that represent different partitions, to improve generalization for the entire networks model, each fold consists of training data, testing data and validation data. Percentage of training data $80 \%$, validation data $10 \%$ and testing data $10 \%$, Since we have dataset consist of 424 records then the training data have 340 records, 42 records for testing data and 42 records for validation data for each fold. For the training data, we have 170 records represent benign diagnosis and 170 malignant diagnoses. And for validation and testing data we have 21 records represent benign diagnosis and 21 malignant diagnoses.

\section{Experimental Results}

In our model, four experiments are done depending on the number of inputs

The following parameters are used:

a) A Feedforward Back-propagation neural network is used for building all models.

b) The number of neurons, in four models, in the input layer are 32, 31, 31, 30 (representing the symptoms before renal failure) 
c) The number of neurons in the output layer is 1 in all models (representing one class of the cause the neural will generate).

d) The training algorithm that was used for training the models is Resilient Backpropagation.

e) The activation function that is used for all the hidden layers is TANH, on the other hand, the activation function that is used in the output layer is LF or TANH (the difference between the two models).

f) The following values were used 0.0000001 , and 6 for the performance goal error and the number of validation checks to avoid the overfitting of the network, respectively.

When starting to build the two models and the four experiments in each model, we started with one hidden layer and ended with 5-6 hidden layers depends on the experiment (Table 1).

Table 1: Table of Hospitals and questionnaires that have been collected.

\begin{tabular}{|c|c|c|c|c|c|c|c|}
\hline Hospital & D.M. & HTN & GN & VUR & Polycystic & D.M/HTN & Total \\
\hline Jordan University Hospital & 0 & 2 & 6 & 5 & 5 & 7 & 25 \\
\hline Aljazeera Hospital & 1 & 4 & 1 & 3 & 1 & 4 & 3 \\
\hline Al Hikma Hospital & 6 & 4 & 13 & 4 & 3 & 33 \\
\hline Al Razi Hospital & 4 & 6 & 1 & 3 & 4 & 7 & 3 \\
\hline Al Zaytouna Hospital & 6 & 4 & 6 & 8 & 9 & 25 \\
\hline Ahmed Hamaideh Hospital & 4 & 5 & 1 & 2 & 2 & 31 \\
\hline Akilah Hospital & 9 & 5 & 2 & 5 & 6 & 4 & 30 \\
\hline Total & 30 & 30 & 30 & 30 & 30 & 30 \\
\hline
\end{tabular}

\section{Model 1 (Linear Activation function)}

In model 1 the linear activation function is used between the last hidden layer and the output layer. Table 2 will show the comparison for experiments in the first model.

Table 2: Comparison of the four experiments.

\begin{tabular}{|c|c|c|c|c|}
\hline Experiment \# & Higher Accuracy & Network Architecture & Lower Accuracy & Network Architecture2 \\
\hline Experiment 1 & $62.22 \%$ & $32-19-9-1$ & $25.56 \%$ & $32-1-1$ \\
\hline Experiment 2 & $67.22 \%$ & $31-15-7-1$ & $22.22 \%$ & $31-1-1-1$ \\
\hline Experiment 3 & $67.78 \%$ & $31-17-9-5-1$ & $23.33 \%$ & $31-1-1-1$ \\
\hline Experiment 4 & $66.10 \%$ & $30-14-7-1$ & $26.10 \%$ & $30-1-1-1$ \\
\hline
\end{tabular}

According to Table, there is not much difference between experiment $2 \& 3$, and this due to the two input variables that are ignored during these experiments. These two inputs are connected, that they are talking about age in general. Considering that the second one is better to use slightly due to some reasons: First one of these diseases are connected to the age as some of them reflect kidneys during a long time while others are not. And due to the difference of age in samples, some diseases affected young people and some are not, which affects the number of years during dialysis. Another reason, diseases like diabetes with lack of care may affect other organs, which lead to death during a small amount of time.

\section{Model 2 (TANH)}

In model 2 TANH activation function is used between the last hidden layer and the output layer. Model 2 did not succeed at all. All of its classification accuracy in the four experiments is within
$40 \%$. Table 2 shows random samples classification accuracy in model 2.

Table 3: samples of classification accuracy for model 2.

\begin{tabular}{|c|c|}
\hline Network Architecture & Classification Accuracy \\
\hline $32-16-1$ & 28.8889 \\
\hline $32-16-8-1$ & 33.8889 \\
\hline $32-16-8-4-1$ & 36.1111 \\
\hline $32-16-8-4-2-1$ & 39.4444 \\
\hline $31-15-1$ & 31.6667 \\
\hline $31-15-7-1$ & 37.7778 \\
\hline $31-15-7-3-1$ & 37.7778 \\
\hline $31-15-7-3-2-1$ & 31.1111 \\
\hline
\end{tabular}

To conclude from previous results of models, the LF model works more properly than TANH model no mater number of 
inputs we use. And experiments are the only way to determine, the best network architecture that can be used to solve a specific problem (Table 3).

\section{Conclusion}

In this research, the maximum result that we reach was about 67 classification accuracy. The result was expected, due to the kind of questionnaire that we used. As we mentioned before this questionnaire is depending on patients and their relative's memory. Note that there are many patients spent on dialysis for about 20 -30 years, and the questionnaire is about what happens before dialysis, which means that patients have to remember things they suffered from 20-30 years ago. Another thing to consider is the age of these patients, very old patients and very young patients do not always focus, this makes us ask their close relatives that makes us face another issue, patients might not be aware of some detailed questions which make us fall in mistake of guessing. Additionally, the experiments do not depend on lab results because most of them are missing.

Finally, to get a system that helps doctors to predict the renal failure cause the percentage of $68 \%$, within 5 minutes and with no lab tests, is somehow a success system. And I hope that this may help doctors to deal with serious situations, in the lake of time.

\section{References}

1. Salah B, Alshraideh M, Beidas R, Hayajneh R (2011) Skin cancer recognition by using a neuro-fuzzy system. Cancer informatics 10 .

2. Qatawneh Z, Alshraideh M, Almasri N, Tahat L, Awidi A (2019) Clinical Decision Support System for Venous Thromboembolism Risk Classification. Applied Computing and Informatics 15(1): 12-18.

3. Bezemer T, De Groot MCH, Blasse E, Ten Berg MJ, Kappen TH, et al. (2019) A Human(e) Factor in Clinical Decision Support Systems. J Med Internet Res 21(3).

4. Al Switi M, Alshraideh B, Alshraideh A, Massad A, Alshraideh M (2019) Treatment of Diabetes Type II Using Genetic Algorithm. International Journal of Online and Biomedical Engineering (iJOE) 15(11): 53-68.

5. Hawamdeh Z, Alshraideh M, Al Ajlouni J, Salah I, Holm M, et al. (2012) Development of a decision support system to predict physicians' rehabilitation protocols for patients with knee osteoarthritis. International Journal of Rehabilitation Research 35(3): 214-219.

6. Ali $\mathrm{H}(2008)$ Self-organizing maps for prediction of kidney dysfunction. $16^{\text {th }}$ Telecommunications Forum (TELFOR), Serbia, Belgrade, pp.25-27.

7. Vahdani P, Alavian S, Aminzadeh Z, Raoufy M, Gharibzadeh S, et al. (2009) Using Artificial Neural Network to Predict Cirrhosis in Patients with Chronic Hepatitis B Infection with Seven Routine Laboratory Findings. Hepatitis Monthly 9(4): 271-275.

8. Turkoglu I, Arslanb A, Ilkayc E (2002) An Expert System for Diagnosis of the Heart Valve Diseases. Expert Systems with Applications 23(3): 229-236.

9. Machado LO (1996) Medical Application of Artificial Neural Networks: Connectionist Models of Survival. Master's thesis, Department of Computer Science, University of Stanford, USA.

10. Magoulas GD, Prentza A (2001) Machine Learning in Medical Applications. The University of Athens. 2049, Athens, Greece, pp. 300307.
11. Mehrotra K, Chilukuri K, Mohan, Ranka S (1996) Elements of Artificial Neural Networks. ( $1^{\text {st }}$ ed), New York: Addison-Wesley Publishing Company, Inc.

12. Mitchell TM (2006) The Discipline of Machine Learning. Carnegie Mellon University. School of Computer Science. Pittsburgh, PA, USA.

13. Sirmani PK, Koti MS (2013) Medical Diagnosis Using Ensemble Classifiers - A Novel Machine- Learning Approach. Journal of Advanced Computing 1(6): 9-27.

14. Taylor CM (1997) Selecting Neural Network Topologies: A Hybrid Approach Combining Genetic Algorithms and Neural Networks. Master Thesis. Southwest Missouri State University.

15. Krose B, Smagt P (1996) An Introduction to Neural Networks. ( $8^{\text {th }}$ ed), Amsterdam: The University of Amsterdam.

16. Adeli H, Zhou Z, Dadmehr N (2003) Analysis of EEG records in an epileptic patient using the wavelet transform. Journal of neuroscience methods 123(1): 69 -87.

17. Peterson C, Rognvaldsson T (1991) An Introduction to Artificial Neural Networks. CERN Summer School of Computing, Cern Yellow Report 92(02): 113-170.

18. Poole D, Mackworth A (2010) Artificial Intelligence Foundation of Computation Agents. Cambridge University Press.

19. Riedmiller M, Braun H (1993) A Direct Adaptive Method for Faster Backpropagation Learning: The RPROP Algorithm. Neural Networks, IEEE International Conference, San Francisco, CA, USA, pp. 586-591.

20. Jung TP, Makeig S, McKeown MJ, Bell AJ, Lee TW, et al. (2001) Imaging brain dynamics using independent component analysis. Proceedings of the IEEE 89(7): 1107-1122.

21. Francesco P, Mattea D, Giulio B, David N, Vito CO, et al. (2016) Clinical decision support system for end-stage kidney disease risk estimation in IgA nephropathy patients. Nephrology Dialysis Transplantation 31(1): 80-86.

22. Behnam M, Mohammad HA, Shing IC (2017) Medical Decision Support System for Disease Diagnosis under Uncertainty. Expert Systems with Applications 88(1): 95-108.

23. Baig MM, Gholam HH, Moqeem AA, Mirza F, Lindén M (2019) Clinical decision support systems in-hospital care using ubiquitous devices: Current issues and challenges. Health Informatics J, 25(3): 1091-1104.

24. Faust O, Acharya UR, MIN LC, Sputh BH (2010) Automatic identification of epileptic and background EEG signals using frequency-domain parameters. International Journal of neural systems 20(2): 159-176.

25. Gabor A, Leach R, Dowla F (1996) Automated seizure detection using a self-organizing neural network. Electroencephalography and Clinical Neurophysiology 99(3): 257-266.

26. Khan Y, Gotman J (2003) Wavelet-based automatic seizure detection in intracerebral electroencephalogram. Clinical Neurophysiology 114(5): 898-908.

27. Chua KC, Chandran V, Acharya UR, Lim C (2011) Application of higherorder spectra to identify epileptic EEG. Journal of medical systems 35(6): 1563-1571.

28. Acharya UR, Sree SV, Chattopadhyay S, Yu W, Ang PCA (2011) Application of recurrence quantification analysis for the automated identification of epileptic EEG signals. International Journal of neural systems 21(3): 199-211.

29. Bhaskar P, Reddy EM (2012) Efficient Machine Learning Approach for identifying Disease-Treatment Semantic Relations from Bio-Medical Sentences. International Journal of Computational Engineering Research 2(5): 1425 -1429. 
30. Brause Rüdiger W (2001) Medical Analysis and Diagnosis by Neural Networks. In Proceedings of the Second International Symposium on Medical Data Analysis London, UK, pp. 1-13.

31. Bylander T, Tate L (2006) Using Validation Sets to Avoid Overfitting in AdaBoost. The University of Texas at San Antonio, San Antonio, TX 78249 USA.

32. Karlik B, Olgac AV (2010) Performance Analysis of Various Activation Function in Generalized MLP Architecture of Neural Network. International Journal of Artificial Intelligence and Expert Systems (IJAE) 1(4): 111-122.

33. Adeli H, Ghosh DS, Dadmehr N (2005) Alzheimer's disease: models of computation and analysis of EEGs. Clinical EEG and Neuroscience 36(3): 131-140.
34. Busatto GF, Diniz BS, Zanetti MV (2008) Voxel-based morphometry in Alzheimer's disease. Expert review of neurotherapeutics 8(11): 16911702 .

35. Al Timemy A, Al Naima F (2010) Comparison of Different Neural Network Approaches for the Prediction of Kidney Dysfunction. International Journal of Biological and Life Sciences 6(2): 84-90.

36. Fisher R, Webber W, Lesser R, Arroyo S, Uematsu S (1992) High-frequency EEG activity at the start of seizures. Journal of clinical neurophysiology: official publication of the American Electroencephalographic Society 9(3): 441-448.

37. Sutton RT, Pincock D, Baumgart DC, Daniel CS, Richard NF, et al. (2020) An overview of clinical decision support systems: benefits, risks, and strategies for success. npj Digit Med 3(17): 1-10.

\section{Your next submission with Juniper Publishers will reach you the below assets}

- Quality Editorial service

- Swift Peer Review

- Reprints availability

- E-prints Service

- Manuscript Podcast for convenient understanding

- Global attainment for your research

- Manuscript accessibility in different formats

( Pdf, E-pub, Full Text, Audio)

- Unceasing customer service

Track the below URL for one-step submission https://juniperpublishers.com/online-submission.php 